\title{
Riscos psicossociais no trabalho no setor público brasileiro: revisão sistemática
}

\author{
Psychosocial risks at work in the Brazilian public sector: systematic review
}

\section{Raianne de Souza Rodrigues ${ }^{1}$, Janine Kieling Monteiro ${ }^{2}$ e Kamila Nunes Pires ${ }^{3}$}

Resumo: $O$ objetivo deste estudo foi analisar estudos empíricos da literatura científica nacional sobre os riscos psicossociais no trabalho relativos ao setor público brasileiro dos últimos dez anos, buscando identificar os riscos psicossociais no trabalho presentes nesse contexto laboral e os danos que eles podem desencadear à saúde dos trabalhadores. Foram selecionados 33 artigos, que foram analisados quanto à autoria-instituição-departamento, publicação, delineamento metodológico e resultados. Verificou-se que os riscos psicossociais no trabalho mais prevalentes no setor público se referem a fatores vinculados à sobrecarga de trabalho e à precariedade das condições de trabalho. Entre os danos à saúde dos trabalhadores, destacaramse danos físicos e psicológicos, necessitando de intervenções organizacionais mais efetivas relativas ao gerenciamento dos riscos psicossociais e à promoção de saúde nesse contexto laboral. Diante dos riscos psicossociais evidenciados no setor público brasileiro, destaca-se a necessidade de maior investimento financeiro e melhoria na gestão de pessoas e nos recursos físicos.

Palavras-chave: Riscos psicossociais no trabalho; Saúde mental; Setor público.
Abstract: The aim of this study was to analyze empirical studies from the national scientific literature on psychosocial risks at work related to the Brazilian public sector in the last ten years, seeking to identify the psychosocial risks at work present in this work context and the damage they can cause to the health of workers. Thirty-three articles were selected, which were analyzed in terms of authorship-institutiondepartment, publication, methodological design and results. It was found that the most prevalent psychosocial risks at work in the public sector refer to factors linked to work overload and precarious working conditions. Among the damages to workers' health, physical and psychological damage stood out, requiring more effective organizational interventions related to the management of psychosocial risks and health promotion in this work context. From the psychosocial risks evidenced in the Brazilian public sector, the need for greater financial investment and improvements in the management of people and physical resources is highlighted.

Keywords: Psychosocial risks at work; Mental health; Public sector.

\footnotetext{
1 Psicóloga pela Universidade Federal do Amazonas (UFAM). Mestra em Psicologia pela UFAM. Psicóloga no Instituto Federal de Educação, Ciência e Tecnologia do Amazonas (IFAM). E-mail: raianne.rodrigues@ifam.edu.br

2 Psicóloga pela Universidade Federal do Rio Grande do Sul (UFRGS). Doutora em Psicologia pela UFRGS. Professora titular na graduação e no Programa de Pós-Graduação em Psicologia na Universidade do Vale do Rio dos Sinos (Unisinos). E-mail: janinekm@unisinos.br

3 Acadêmica de Psicologia na Unisinos. E-mail: kmilanp@gmail.com
} 


\section{Introdução}

Para a Organização Internacional do Trabalho (ILO, 2020), os riscos psicossociais no trabalho são fatores resultantes das interações entre questões do trabalho e questões do indivíduo, que impactam no desempenho e na saúde do trabalhador. De acordo com a Organização Mundial da Saúde (WHO, 2015), o ambiente do trabalho tem se tornado um dos principais causadores de doenças mentais, cujas estatísticas apontam que uma a cada cinco pessoas no trabalho podem sofrer de algum problema de saúde mental.

Conhecer os riscos psicossociais a que os trabalhadores estão expostos se faz necessário para identificar e compreender as condiçōes de trabalho nas organizações, a interação entre 0 trabalho e os indivíduos, a satisfação no trabalho e o contexto laboral. Tudo isso, articulado às necessidades do trabalhador, às suas capacidades, à situação pessoal fora do trabalho, à sua inserção cultural e às experiências e vivências dos trabalhadores, conforme apontam Rodrigues, Faiad \& Facas, 2020.

As relaçôes entre o trabalhador e os processos de trabalho vêm sofrendo modificações ao longo dos anos, especialmente na esfera pública. Klein Júnior (2020) afirma que o setor público brasileiro tem estado em permanente pressão do governo e da sociedade para responder às demandas dos cidadãos eà crescente complexidade de seus ambientes, com destaque para a oferta de produtos e serviços de boa qualidade. Acrescenta-se a essa questão, segundo Bichet \& Vargas (2021), o fato de que esses trabalhadores também enfrentam um desmonte dos seus direitos no funcionalismo público brasileiro, que por sua vez, influencia diretamente na sua produtividade e impacta de forma danosa sua saúde.

Diante disso, tem-se como objetivo analisar estudos empíricos da literatura científica nacional sobre os riscos psicossociais no trabalho relativos ao setor público brasileiro dos últimos dez anos (abril de 2011 a abril de
2021). Nesse sentido, buscou-se identificar os riscos psicossociais no trabalho presentes nesse contexto laboral e os danos que eles podem desencadear à saúde dos trabalhadores.

\section{Método}

Com o intuito de atingir os objetivos propostos, realizaram-se buscas dos estudos indexados nas bases de dados Biblioteca Virtual em Saúde (BVS) e Portal de Periódicos CAPES, no mês de abril de 2021. Para consultar os artigos nessas bases de dados, que disponibilizam acesso fácil a estudos nacionais e internacionais, utilizou-se os seguintes descritores em português: "riscos psicossociais no trabalho" OR "fatores psicossociais de risco no trabalho" AND "saúde mental"AND"setor público".

Foram definidos como critérios de inclusão: pesquisas empíricas realizadas no Brasil, mesmo que publicadas internacionalmente; abordar 0 tema riscos psicossociais no trabalho no setor público; estudos publicados nos últimos dez anos (entre abril de 2011 e abril de 2021). Como critérios de exclusão, foram utilizados: teses e dissertações; revisões sistemáticas; artigos teóricos; publicações com data anterior a abril de 2011; pesquisas realizadas em outros países.

No total, encontrou-se 166 estudos na busca inicial, sendo 144 na base de dados da BVS e 22 no Portal de Periódicos CAPES. Os registros foram analisados manualmente, resultando na exclusão de 111 artigos por não se encaixarem nos critérios propostos, terem publicações duplicadas nas bases de dados consultadas ou estarem com o texto completo indisponível. Dessa forma, foram incluídos 33 artigos para este estudo, conforme ilustrado na Figura 1. Ressalta-se que este estudo utilizou as diretrizes do Preferred Reporting Items for Systematic Reviews and Meta-Analyses (PRISMA).

Figura 1 - Fluxograma do processo de inclusão de artigos.

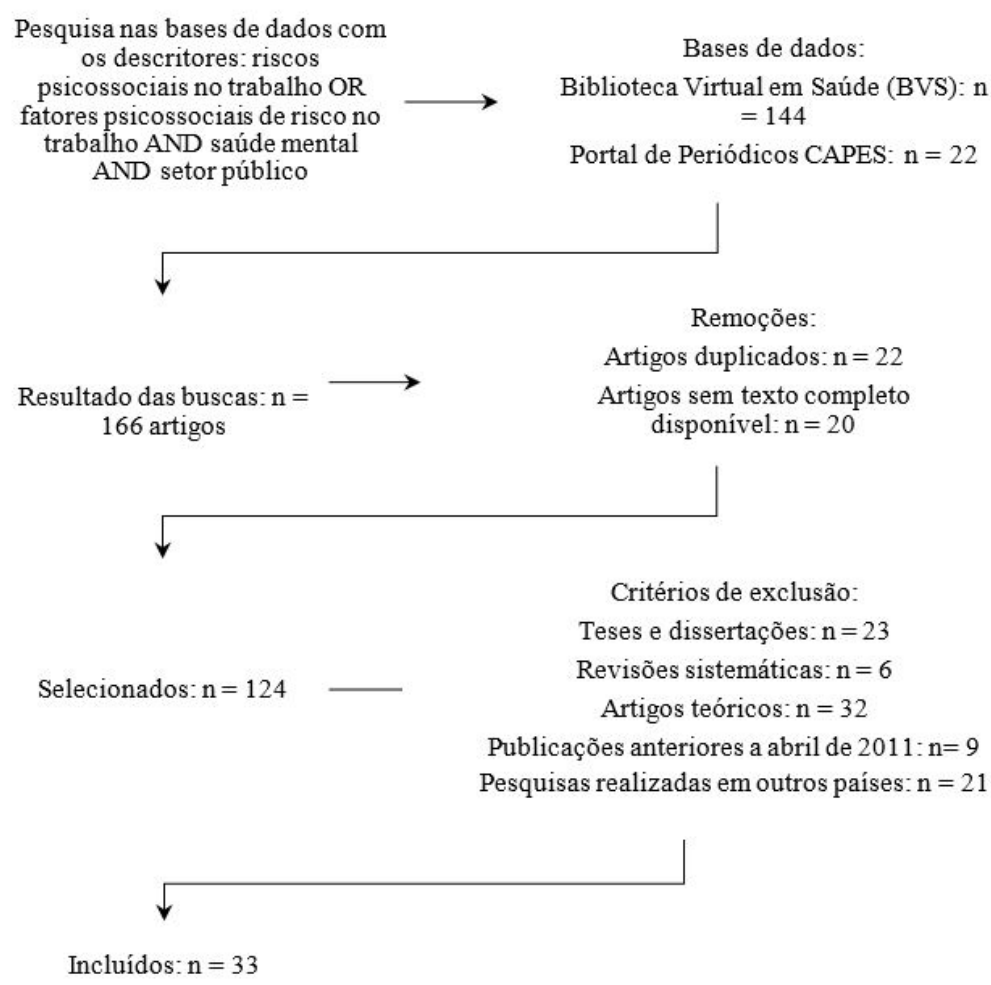


A análise dos artigos foi realizada a partir da leitura dos resumos e do texto na íntegra dos artigos incluídos, buscando as informações necessárias para atender ao objetivo central desta revisão sistemática. Nesse sentido, para construir as categorias de análise, o presente estudo cumpriu as seguintes etapas: leitura detalhada de cada artigo; realização de resumos; síntese focada nos resultados dos estudos. A seguir estão descritas as categorias propostas: Autoria-Instituição-Departamento: número de autores, afiliação institucional da maioria dos autores, departamento ou programa do curso de vinculação dos autores; Publicação: ano e periódico no qual 0 artigo foi publicado; Delineamento metodológico: tipo de pesquisa, participantes (categoria profissional e tamanho da amostra) e instrumentos; Resultados: identificação dos principais riscos psicossociais no trabalho e os danos que esses riscos podem desencadear à saúde dos trabalhadores.

\section{Resultados e discussão}

Os 33 artigos selecionados estão descritos na Tabela 1, com informações do título, autoria dos estudos e ano de publicação. Os achados desses estudos sobre os riscos psicossociais no trabalho no setor público e os danos à saúde dos trabalhadores serão descritos por meio de tabelas ou de forma textual.

Tabela 1 - Artigos incluídos.

\begin{tabular}{|c|c|c|c|}
\hline No & Título & Autoria & Ano \\
\hline 1 & $\begin{array}{l}\begin{array}{c}\text { Burnout syndrome and associated factors among health professionals of a } \\
\text { public hospital }\end{array} \\
\end{array}$ & Ebling \& Carlotto & 2012 \\
\hline 2 & $\begin{array}{l}\text { Condiçôes de Trabalho e Morbidade Referida de Policiais Militares, Recife- } \\
\text {-PE, Brasil }\end{array}$ & Ferreira, Bonfim \& Augusto & 2012 \\
\hline 3 & $\begin{array}{l}\text { Fatores associados ao sofrimento psíquico de policiais militares da cidade } \\
\text { do Rio de Janeiro, Brasil }\end{array}$ & Souza, Minayo, Silva \& Pires & 2012 \\
\hline 4 & $\begin{array}{l}\text { Riscos Psicossociais e Incapacidade do Servidor Público: Um Estudo de } \\
\text { Caso }\end{array}$ & Serafim, Campos, Cruz \& Rabuske & 2012 \\
\hline 5 & $\begin{array}{l}\text { Riscos psicossociais em equipes de saúde da família: carga, ritmo e } \\
\text { esquema de trabalho }\end{array}$ & Camelo, Chaves, Silva \& Angerami & 2012 \\
\hline 6 & $\begin{array}{c}\text { A situação de trabalho da enfermagem e os riscos ocupacionais na } \\
\text { perspectiva da ergologia }\end{array}$ & Fontana \& Lautert & 2013 \\
\hline 7 & $\begin{array}{l}\text { Fatores de risco psicossocial em terapia intensiva neonatal: repercussões } \\
\text { para a saúde do enfermeiro }\end{array}$ & $\begin{array}{l}\text { Oliveira, Silva, Perez Junior, Costa, Nascimen- } \\
\text { to \& Souza }\end{array}$ & 2013 \\
\hline 8 & $\begin{array}{l}\text { Gerenciamento de riscos e segurança no trabalho em unidades de saúde } \\
\text { da família }\end{array}$ & Medeiros, Costa, Sousa \& Rosenstock & 2013 \\
\hline 9 & $\begin{array}{c}\text { Riscos ocupacionais e problemas de saúde percebidos por trabalhadores de } \\
\text { enfermagem em unidade hospitalar }\end{array}$ & Beleza, Gouveia, Robazzi, Torres \& Azevedo & 2013 \\
\hline 10 & $\begin{array}{c}\text { Riscos ocupacionais e agravos à saúde dos trabalhadores em uma unidade } \\
\text { ambulatorial especializada }\end{array}$ & $\begin{array}{l}\text { Souza, Pires, Gonçalves, Cunha, Ribeiro \& } \\
\text { Vieira }\end{array}$ & 2014 \\
\hline 11 & $\begin{array}{l}\text { A síndrome de burnout em profissionais da Rede de Atenção Primária à } \\
\text { Saúde de Aracaju, Brasil }\end{array}$ & $\begin{array}{l}\text { Silva, Nunes, Santana, Reis, Machado Neto } \\
\text { \& Lima }\end{array}$ & 2015 \\
\hline 12 & $\begin{array}{l}0 \text { trabalho de enfermagem em centro de tratamento de queimados: riscos } \\
\text { psicossociais }\end{array}$ & Oliveira, Guerra, Almeida, Silva, Fabri \& Vieira & 2015 \\
\hline 13 & 0 trabalho no cárcere: reflexões acerca da saúde do agente penitenciário & Jaskowiak \& Fontana & 2015 \\
\hline 14 & $\begin{array}{l}\text { Riscos psicossociais em unidades especializadas: implicações para a forma- } \\
\text { ção e a saúde do enfermeiro residente }\end{array}$ & $\begin{array}{l}\text { Lima, Oliveira, Mauro, Lisboa, Assad \& } \\
\text { Carvalho }\end{array}$ & 2015 \\
\hline 15 & $\begin{array}{l}\text { Seleção, capacitação e formação da equipe de profissionais dos abrigos: } 0 \\
\text { hiato entre o prescrito e o real }\end{array}$ & Halpern, Leite \& Moraes & 2015 \\
\hline 16 & $\begin{array}{l}\text { Vivências de prazer e sofrimento mental em um Centro de Atenção } \\
\text { Psicossocial }\end{array}$ & Azevedo \& Figueredo & 2015 \\
\hline 17 & Occupational Stress and Quality of Life in Nursing & Silva \& Guimarães & 2016 \\
\hline 18 & $\begin{array}{l}\text { Riscos ocupacionais e adoecimentos entre professores da rede municipal } \\
\text { de ensino }\end{array}$ & $\begin{array}{l}\text { Silva, Fritsch, Dalri, Giulena, Silveira, Evange- } \\
\text { lista, Pelazza \& Maia }\end{array}$ & 2016 \\
\hline 19 & $\begin{array}{l}\text { Risco ocupacional entre profissionais de enfermagem de setores críticos e } \\
\text { adesão a precaução padrão }\end{array}$ & Batista, Moura, Sousa \& Andrade & 2017 \\
\hline
\end{tabular}




\begin{tabular}{|c|c|c|c|}
\hline No & Título & Autoria & Ano \\
\hline 20 & Burnout e a organização do trabalho na Enfermagem & Sobral, Stephan, Bedin-Zanatta \& De-Lucca & 2018 \\
\hline 21 & $\begin{array}{l}\text { Cultura de segurança: percepção dos profissionais de saúde em um } \\
\text { hospital mental }\end{array}$ & $\begin{array}{l}\text { Oliveira, Cavalcante, Aires, Freitas, Silva, } \\
\text { Marinho \& Carvalho }\end{array}$ & 2018 \\
\hline 22 & $\begin{array}{l}\text { Demandas psicológicas e controle do processo de trabalho de servidores } \\
\text { de uma universidade pública }\end{array}$ & $\begin{array}{l}\text { Moura, Greco, Paschoalin, Portela, Arreguy- } \\
\text {-Sena \& Chaoubah }\end{array}$ & 2018 \\
\hline 23 & $\begin{array}{l}\text { Estresse ocupacional e fatores associados em servidores públicos de uma } \\
\text { universidade federal do sul do Brasil }\end{array}$ & Lopes \& Silva & 2018 \\
\hline 24 & Saúde mental e trabalho docente & Moreira \& Rodrigues & 2018 \\
\hline 25 & $\begin{array}{l}\text { Fatores psicossociais e condições ambientais: sua influência na capacidade } \\
\text { para o trabalho na área da assistência social }\end{array}$ & $\begin{array}{l}\text { Roncoleta, Jorge, Maríngolo, Justino \& } \\
\text { Silva-e-Dutra }\end{array}$ & 2019 \\
\hline 26 & Mapeamento dos Riscos Psicossociais no SAMU/DF & Araujo \& Oliveira & 2019 \\
\hline 27 & $\begin{array}{l}\text { Reconhecimento dos riscos no trabalho do Consultório na Rua: um } \\
\text { processo participativo }\end{array}$ & $\begin{array}{l}\text { Lima, Almeida, Costa, Marques, Lima Júnior } \\
\qquad \& \text { Rocha }\end{array}$ & 2019 \\
\hline 28 & $\begin{array}{l}\text { Riscos psicossociais relacionados ao enfermeiro no hospital psiquíátrico e } \\
\text { estratégias de gestão }\end{array}$ & Scozzafave, Leal, Soares \& Henriques & 2019 \\
\hline 29 & $\begin{array}{l}\text { Fatores associados aos riscos de adoecimento da equipe de enfermagem } \\
\text { no trabalho em instituiçãa psiquiátrica }\end{array}$ & $\begin{array}{c}\text { Sousa, Zeitoune, Portela, Tracera, Moraes \& } \\
\text { Figueiró }\end{array}$ & 2020 \\
\hline 30 & $\begin{array}{l}\text { Riscos psicossociais no trabalho em programas de pós-graduação stricto } \\
\text { sensu públicos e privados }\end{array}$ & Rissi, Monteiro \& Ferreira & 2020 \\
\hline 31 & $\begin{array}{l}\text { Riscos psicossociais relacionados ao trabalho do enfermeiro da Saúde da } \\
\text { Família e estratégias de gerenciamento }\end{array}$ & Celestino, Leal, Lopes \& Henriques & 2020 \\
\hline 32 & $\begin{array}{l}\text { Trabalho da enfermagem no centro cirúrgico e os riscos psicossociais } \\
\text { relacionados aos modos de gestão }\end{array}$ & Madrid, Kotekewis \& Glanzner & 2020 \\
\hline 33 & $\begin{array}{l}\text { Trabalho e saúde: a perspectiva dos agentes de combate a endemias do } \\
\text { município de Belo Horizonte, MG }\end{array}$ & Matos, Silva \& Silveira & 2020 \\
\hline
\end{tabular}

Em relação ao número de autores por estudo, foi constatado que prevaleceram artigos redigidos por 2 ou 4 autores, o que parece indicar que as produções são originadas de resultados obtidos em grupos de pesquisa. Quanto ao ano das publicações, verificou-se que não houve registro de publicações no ano de 2011, bem como a maior frequência foi registrada em 2015, com 6 publicações, seguida pelos anos de 2012, 2018 e 2020, com 5 publicações em cada ano. Tais indicadores demonstram que as publicações de estudos sobre os riscos psicossociais no trabal ho no setor público vêm indicando uma tendência de crescimento ao longo dos últimos anos.

No que concerne à afiliação institucional da maioria dos autores ou do autor principal de cada artigo, foi identificado que a maior parte dos estudos (4) foi realizada por pesquisadores vinculados a Universidade do Estado do Rio de Janeiro (UERJ), seguido pela Universidade de São Paulo (USP) (3) e Universidade Federal do Rio Grande do Sul (UFRGS) (3), o que sugere que a preponderância dos estudos sobre riscos psicossociais no trabalho no setor público se concentra na Região Sudeste, seguida pela Região Sul. Quanto ao tipo de instituição, constatou-se que a maioria das produções científicas (29) foi desenvolvida por autores vinculados a universidades públicas.

Os departamentos ou programas da universidade, aos quais os autores estão vinculados, mais prevalentes foram das Ciências da Saúde, com predomínio da área de Enfermagem (16), que corresponde à metade das produções analisadas, seguida pela Psicologia (4), cuja representatividade ainda é incipiente nas pesquisas sobre os fatores psicossociais de risco no trabalho. Cumpre salientar que, embora o resultado aponte a preponderância da Enfermagem, há também que se considerar que paulatinamente outras áreas estão começando a privilegiar estudos com foco na saúde do trabalhador, indicando uma abordagem interdisciplinar sobre os riscos psicossociais no trabalho no setor público.

Quanto aos periódicos em que os artigos foram publicados, a Tabela 2 demonstra que a maioria dos estudos (28) foram indexados em periódicos do Brasil. Verificou-se ainda que a prevalência das publicações foi em periódicos da área de Enfermagem. 
Tabela 2 - Periódicos de publicação dos artigos.

\begin{tabular}{|c|c|c|}
\hline Periódico & Frequência absoluta & Frequência relativa (\%) \\
\hline Revista Brasileira de Enfermagem & $\mathbf{3}$ & $\mathbf{9}$ \\
\hline Revista de Enfermagem da UERJ & $\mathbf{3}$ & $\mathbf{9}$ \\
\hline Ciência \& Saúde Coletiva & $\mathbf{3}$ & $\mathbf{9}$ \\
\hline Psicologia Ciência e Profissão & 2 & 6,2 \\
\hline Revista da Escola de Enfermagem da USP & 2 & 6,2 \\
\hline Revista Latino-Americana de Enfermagem & 2 & 6,2 \\
\hline Revista Brasileira de Medicina do Trabalho & 2 & 3 \\
\hline Cadernos de Saúde Pública & 1 & 3 \\
\hline Ciência y Enfermaría & 1 & 3 \\
\hline Estudos de Psicologia & 1 & 3 \\
\hline Journal Health NPEPS & 1 & 3 \\
\hline Journal of Rescarch Fundamental Care Online & 1 & 3 \\
\hline Paidéía & 1 & 3 \\
\hline Revista Brasileira de Ciências da Saúde & 1 & 3 \\
\hline Revista Brasileira de Saúde Ocupacional & 1 & 3 \\
\hline Revista Cubana de Enfermaría & 1 & 3 \\
\hline Revista Gaúcha de Enfermagem & 1 & 3 \\
\hline Revista Mineira de Enfermagem & 1 & 3 \\
\hline Revista Psicologia Organização e Trabalho & 1 & 3 \\
\hline Saúde \& Sociedade & 1 & 3 \\
\hline Trabalho, Educação e Saúde & 1 & 3 \\
\hline Trabalho En(Cena) & $\mathbf{1 0 0}$ & \\
\hline Trends in Psychiatry and Psychotherapy & 1 & 3 \\
\hline Total & 1 & 3 \\
\hline
\end{tabular}

Todos os estudos analisados foram de coorte transversal. Quanto ao tipo de pesquisa adotado, ilustrado na Tabela 3, houve predomínio de estudos quantitativos (17) embora notou-se a incipiente utilização de pesquisas multimétodos na investigação dos riscos psicossociais no trabalho, o que sugere a preocupação dos pesquisadores em não somente avaliar esses fenômenos, mas também compreendê-los de uma forma mais aprofundada, possibilitando ao trabal hador um espaço de fala e escuta sobre suas vivências relacionadas ao trabalho.

Tabela 3 - Tipo de Pesquisa adotado nos estudos

\begin{tabular}{|c|c|c|}
\hline Tipo de Pesquisa & Frequência absoluta & Frequência relativa (\%) \\
\hline Quantitativa & $\mathbf{1 7}$ & $\mathbf{5 1 , 7}$ \\
\hline Qualitativa & 13 & 39,3 \\
\hline Mista (Qualitativa e Quantitativa) & 3 & 9 \\
\hline Total & $\mathbf{3 3}$ & $\mathbf{1 0 0}$ \\
\hline
\end{tabular}

As categorias profissionais de participantes, descritas na Tabela 4, apontam representatividade maior para trabalhadores da área da saúde (21), especialmente os profissionais de enfermagem, que totalizaram 12 participa- ções nas pesquisas. No que concerne ao tamanho da amostra das pesquisas, conforme demonstra a Tabela 5, variou de 1 a 1.120, com predomínio de $\leq$ 200 participantes. 
Tabela 4 - Categoria profissional dos participantes.

\begin{tabular}{|c|c|c|}
\hline Categoria profissional dos participantes & Frequência absoluta & Frequência relativa (\%) \\
\hline Profissionais da saúde & $\mathbf{2 1}$ & $\mathbf{6 3 , 9}$ \\
\hline Profissionais da educação & 5 & 15,1 \\
\hline Profissionais da assistência social & 3 & 9 \\
\hline Agentes da segurança pública & 3 & 9 \\
\hline Agente de Serviços Gerais & 1 & 3 \\
\hline Total & $\mathbf{3 3}$ & $\mathbf{1 0 0}$ \\
\hline
\end{tabular}

Tabela 5 - Tamanho da amostra das pesquisas

\begin{tabular}{|c|c|c|}
\hline Tamanho da amostra & Frequência absoluta & Frequência relativa (\%) \\
\hline$\leq 200$ & 25 & 75,7 \\
\hline $201-400$ & 6 & 18,1 \\
\hline$\geq 401$ & 2 & 6,2 \\
\hline Total & $\mathbf{3 3}$ & $\mathbf{1 0 0}$ \\
\hline
\end{tabular}

Em relação aos instrumentos utilizados nos estudos, houve prevalência de questionários e escalas de avaliação dos fatores psicossociais no trabalho e rastreio da saúde mental do trabalhador nas pesquisas de abordagem quantitativa, com destaque para o Job Content Questionnaire (JCQ) ou Job Stress Scale (JSS) (4), Protocolo de Avaliação dos Riscos Psicossociais no Trabalho (PROART) (3), Maslach Burnout Invetory (MBI) (3) e o Self-Reporting Questionnaire (SRQ-20) (2). Nas pesquisas de abordagem qualitativa, por sua vez, teve predominância de entrevistas (16) e grupos focais (3).

Quanto aos resultados desses estudos, verificou-se que os riscos psicossociais no trabalho no setor público mais prevalentes se referem a fatores vinculados à avaliação das vivências de sofrimento, as quais incluem a interação entre questões do trabalhador e questões do trabalho (Camelo et al., 2012; Serafim et al., 2012; Azevedo \& Figueredo, 2015; Araujo \& Oliveira , 2019; Scozzafave et al, 2019; Celestino et al., 2020; Matos, Silva \& Silveira, 2020; Rissi, Monteiro \& Ferreira, 2020). A sobrecarga (longas jornadas de trabalho, pressões por produtividade e ritmo de trabalho intenso) e as condições precárias de trabalho (falta de recursos materiais ou manutenção de equipamentos, espaço físico inadequado e insuficiência no número de trabalhadores) foram os riscos psicossociais mais citados nos estudos analisados, especialmente os que envolveram profissionais da área de saúde (Madrid, Kotekewis \& Glanzner, 2020; Sousa et al., 2020; 0 liveira et al., 2018; Sobral et al., 2018; Silva et al., 2015; Medeiros et al., 2014; Souza et al., 2014; Beleza et al., 2013).

Outro dado preocupante revelado nas pesquisas se refere à violência no trabalho. 0 tipo de ocorrência prevalente foi a violência psicológica, apontada nos estudos com profissionais da saúde (Celestino et al., 2020; Matos, Silva \& Silveira, 2020; Scozzafave et al., 2019; Fontana \& Lautert, 2013), profissionais da assistência social (Lima et al., 2019; Roncoleta et al., 2019; Halpern, Leite \& Moraes, 2015), profissionais da educação (Moreira \& Rodrigues, 2018) e agente de serviços gerais (Serafim et al., 2012).

A falta de autonomia na realização das tarefas ou na participação nas decisões, bem como os conflitos de relacionamento foram fatores de destaque nos estudos com trabalhadores de enfermagem (Celestino et al., 2020; Madrid, Kotekewis \& Glanzner, 2020; Sousa et al., 2020; Scozzafave et al., 2019; Sobral et al., 2018; Silva \& Guimarães, 2016; Lima et al., 2015), agentes de combates a endemias (Matos, Silva \& Silveira, 2020) e policiais militares (Ferreira, Bonfim \& Augusto, 2012). Somado a isso, a insatisfação profissional também foi mais marcante nos estudos com profissionais da área da saúde (Matos, Silva \& Silveira, 2020; Silva et al, 2015), embora também seja vivenciada por profissionais da educação (Greco et al., 2018), profissionais da segurança pública (Jaskowiak \& Fontana, 2015) e profissionais da assistência social (Halpern, Leite \& Moraes, 2015).

Ressalva-se que outros riscos psicossociais no trabalho foram apontados nos estudos analisados. Dentre eles, a falta de reconhecimento (Rissi, Monteiro \& Ferreira, 2020; Sousa et al., 2020; Azevedo \& Figueredo, 2015), a desmotivação e o conflito entre as exigências do lar e do trabalho (Celestino et al., 2020; Scozzafave et al., 2019) e a rigidez na estrutura hierárquica no ambiente laboral (Madrid, Kotekewis \& Glanzner, 2020; Araujo \& Oliveira, 2019).

Em relação aos danos à saúde dos trabalhadores decorrentes da exposição aos riscos psicossociais no trabalho no setor público, houve prevalência de danos físicos e psicológicos em relação aos danos sociais. Os danos físicos mais citados foram dor de cabeça, dores nas costas, dores no corpo, dores no braço, distúrbios circulatórios, problemas digestivos, alterações no sono e doenças osteomusculares (Matos, Silva \& Silveira, 2020; Rissi, Monteiro \& Ferreira, 2020; Sousa et al., 2020; Araujo \& Oliveira, 2019; Moura et al., 2018; Batista, Moura, Sousa \& Andrade, 2017; Silva et al., 2016; Azevedo \& Figueredo, 2015; Souza et al., 2014; Beleza et al., 2013; Fontana \& Lautert, 2013; Souza et al., 2012).

No que se refere aos danos psicológicos, destacaram-se o estresse e a Síndrome de Burnout (Sousa et al., 2020; Lopes \& Silva, 2018; Oliveira et al., 2018; Sobral et al., 2018; Silva \& Guimarães, 2016; Lima et al., 2015; Oliveira et al., 2015; Silva et al., 2015; Fontana \& Lautert, 2013; Oliveira et al, 2013; Ebling \& (arlotto, 2012; Souza et al., 2012), seguida por transtornos de ansiedade e depressão (Roncoleta et al., 2019; Scozzafave et al., 2019; Moreira \& Rodrigues, 2018; Moura et al., 2018; Medeiros et al., 2014; Beleza et al., 2013; Ferreira, Bonfim \& Augusto, 2012; Serafim et al., 2012). Já os danos sociais foram apontados somente no estudo de Rissi, Monteiro \& Ferreira (2020) 
com professores da pós-graduação de universidades públicas e privadas, cujo destaque foi para a impaciência com as pessoas em geral, agressividade nos relacionamentos interpessoais e insensibilidade em relação aos colegas.

\section{Considerações finais}

0 estudo bibliométrico possibilitou identificar características relevantes dos estudos sobre os riscos psicossociais no trabalho no setor público brasileiro, assim como al gumas lacunas. Entre as principais características, destacaram-se a centralização da produção científica nacional sobre os riscos psicossociais no trabalho no setor público na área da saúde, com ênfase à Enfermagem, que também detém o predomínio da divulgação dos estudos em periódicos da área.

Outros pontos pertinentes referem-se à concentração geográfica na Região Sudeste da autoria das pesquisas, proveniente em sua maioria de universidades públicas, através do delineamento metodológico de abordagem quantitativa, com profissionais da área de saúde. Verificou-se que os riscos psicossociais no trabalho mais prevalentes no setor público se referem a fatores vinculados à sobrecarga de trabalho e precariedade das condições de trabalho, causando danos significativos à saúde dos servidores.

Dentre as lacunas, verificou-se a falta de estudos longitudinais e a incipiente produção de pesquisas sobre os riscos psicossociais no trabalho no setor público por outras áreas, especialmente a Psicologia. Além disso, outro gargalo reside justamente na escassez de estudos sobre os fatores psicossociais de risco no trabalho e seus desfechos na saúde de trabalhadores nos diferentes setores do âmbito público, a saber, assistência social, segurança e educação (especialmente o ensino profissional e tecnológico), pouco investigados nos estudos analisados nesta revisão.

Diante dos riscos psicossociais evidenciados no setor público brasileiro, destaca-se a necessidade de maior investimento financeiro e melhorias na gestão de pessoas (contratação e capacitação de pessoal) e nos recursos físicos (estrutura, materiais e equipamentos para 0 trabalho). Recomenda-se para futuros estudos: realização de pesquisas de coorte longitudinal; abordagem do tema como objeto de estudo em uma perspectiva interdisciplinar, onde a Psicologia desponte como uma área que possa privilegiar tais estudos; maior diversidade de categorias profissionais a serem investigadas. Outro sugestão refere-se à realização de um estudo bibliométrico que abarque a literatura internacional, de forma a subsidiar comparações entre os riscos psicossociais no trabalho no setor público brasileiro e em outros países, identificando semelhanças e diferenças.

\section{Referências}

Araujo, L. K. R., \& Oliveira, S. S. (2019). Mapeamento dos Riscos Psicossociais no SAMU/DF. Psicologia: Ciência e Profissão, 39, e184126. doi: https://doi. org/10.1590/1982-3703003184126.

Azevedo, A. P. F., \& Figueredo, V. C. N. (2015). Vivências de prazer e sofrimento mental em um Centro de Atenção Psicossocial. Revista Psicologia Organizações e Trabalho, 15(1), 30-42. doi: https://dx.doi.org/10.17652/ rpot/2015.1.431.

Batista, 0. M. A, Moura, M. E. B., Sousa, A. F. L., \& Andrade, D. (2017). Risco ocupacional entre profissionais de enfermagem de setores críticos e adesão a precaução padrão. Revista (ubana de Enfermería, 33(3), e1169. Recuperado de http://scielo.sld.cu/scielo.php?script=sci_arttext\&pi$d=$ S0864-03192017000300011\&lng=es\&tlng=pt.
Beleza, C. M. F., Gouveia, M. T. O., Robazzi, M. L. C. C., Torres, C. R. D., \& Azevedo, G. A. V. (2013). Riscos ocupacionais e problemas de saúde percebidos por trabalhadores de enfermagem em unidade hospitalar. Ciencia y enfermería, 19(3), 63-71. doi: https://dx.doi.org/10.4067/ S0717-95532013000300008.

Bichet, M., \& Vargas, S. M. L. (2021). Fatores influenciadores na satisfação e motivação no trabalho no setor público municipal. Desafio Online, 9(1), 138-162. Recuperado de https://desafioonline.ufms.br/index.php/deson/ article/view/10195.

Camelo, S. H. H., Chaves, L, D. P., Silva, V. L. S., \& Angerami, E. L. S. (2012). Riscos psicossociais em equipes de saúde da família: carga, ritmo e esquema de trabalho. Revista de Enfermagem da UERJ, 20(6), 733-739. Recuperado de https://www.e-publicacoes.uerj.br/index.php/enfermagemuerj/ article/view/5990.

Celestino, L. C., Leal, L. A., Lopes, O. C. A., \& Henriques, S. H.. (2020). Riscos psicossociais relacionados ao trabalho do enfermeiro da Saúde da Família e estratégias de gerenciamento. Revista da Escola de Enfermagem da USP, 54, e03602. doi: https://doi.org/10.1590/s1980-220×2018055603602.

Ebling, M., \& Carlotto, M. S. (2012). Burnout syndrome and associated factors among health professionals of a public hospital. Trends in Psychiatry and Psychotherapy, 34(2), 93-100. doi: https://dx.doi.org/10.1590/S223760892012000200008.

Ferreira, D. K. S., Bonfim, C., \& Augusto, L. G. S. (2012). Condições de Trabalho e Morbidade Referida de Policiais Militares, Recife-PE, Brasil. Saúde \& Sociedade, 21(4), 989-1000. Recuperado de https://www.scielo.br/pdf/ sausoc/v21n4/v21n4a16.pdf.

Fontana, R. T., \& Lautert, L. (2013). A situação de trabalho da enfermagem e os riscos ocupacionais na perspectiva da ergologia. Revista Latino-Americana de Enfermagem, 21(6), 1306-1313. doi: https://doi.org/10.1590/01041169.3105 .2368 .

Halpern, E. E., Leite, L. M. C., \& Moraes, M. C. M. B. (2015). Abrigo seleção profissional, capacitação e treinamento: a abrangência entre o que é prescrito e a realidade. Trabalho, Educação e Saúde, 13 (Supl. 1), 91-113. doi; https://dx.doi.org/10.1590/1981-7746-sip00033.

International Labour Organization - ILO (2020). Managing work-related psychosocial risks during the COVID-19 pandemic - Geneva: ILO 2020. Recuperado de https://www.ilo.org/global/topics/safety-and-health-at-work/ resources-library/publications/WCMS_748638/lang--en/index.htm.

Jaskowiak, C. R., \& Fontana, R. T. (2015). 0 trabalho no cárcere: reflexões acerca da saúde do agente penitenciário. Revista Brasileira de Enfermagem, 68(2), 235-243. doi: https://doi.org/10.1590/0034-7167.2015680208i.

Klein Júnior, V. H. (2020). Gestão de riscos no setor público brasileiro: uma nova lógica de accountability. Revista de Contabilidade e Organizações, 14, e163964. doi: http://dx.doi.org/10.11606/issn.1982-6486. rco.2020.163964.

Lima, A. F. S., Almeida, L. W. S., Costa, L. M. C., Marques, E. S., Lima Júnior, M. C. F., \& Rocha, K. R. S. L. (2019). Reconhecimento dos riscos no trabalho do Consultório na Rua: um processo participativo. Revista da Escola de Enfermagem da USP, 53, e03495. doi: https://dx.doi.org/10.1590/s1980$220 \times 2018022603495$.

Lima, L.S. V., Oliveira, E. B., Mauro, M. Y. C., Lisboa, M. T. L., Assad, L. G., \& Carvalho, R. A. C. (2015). Riscos psicossociais em unidades especializadas: implicações para a formação e a saúde do enfermeiro residente. Revista de Enfermagem da UERJ, 23(2), 229-234. doi: https://doi.org/10.12957/ reuerj.2015.16507.

Lopes, S.V., \& Silva, M. C. (2018). Estresse ocupacional e fatores associados em servidores públicos de uma universidade federal do sul do Brasil. Ciência \& Saúde Coletiva, 23(11), 3869-3880. doi: https://doi.org/10.1590/1413812320182311.28682015 . 
Madrid, B. P., Kotekewis, K., \& Glanzner, C. H. (2020). Trabalho da enfermagem no centro cirúrgico e os riscos psicossociais relacionados aos modos de gestão. Revista Gaúcha de Enfermagem, 41, e20190447. doi: https://doi. org/10.1590/1983-1447.2020.20190447.

Matos, G. C. R., Silva, J. M., \& Silveira, A. M. (2020). Trabalho e saúde: a perspectiva dos agentes de combate a endemias do município de Belo Horizonte, MG. Revista Brasileira de Saúde Ocupacional, 45, e15. doi: https://doi.org/10.1590/2317-6369000031117.

Medeiros, A. L., Costa, M. B. S., Sousa, M. C. J., \& Rosenstock, K. I. V. (2014). Gerenciamento de riscos e segurança no trabalho em unidades de saúde da família. Revista Brasileira De Ciências Da Saúde, 17(4), 341-348. Recuperado de https://periodicos.ufpb.br/ojs/index.php/rbcs/article/view/12677.

Moreira, D. Z., \& Rodrigues, M. B. (2018). Saúde mental e trabalho docente. Estudos de Psicologia, 23(3), 236-247. Recuperado de http://pepsic. bvsalud.org/pdf/epsic/v23n3/a04v23n3.pdf

Moura, D. C. A., Greco, R. M., Paschoalin, H. C., Portela, L. F., Arreguy-Sena, C., \& Chaoubah, A. (2018). Demandas psicológicas e controle do processo de trabalho de servidores de uma universidade pública. Ciência \& Saúde Coletiva, 23(2), 481-490. doi: https://doi.org/10.1590/141381232018232.13892015 .

Oliveira, E. B., Guerra, O. A., Almeida, F. P. F. M., Silva, A. V., Fabri, J. M. G., Vieira, M. L. C. (2015). The nursing work at an burn center: psychosocial risks. Journal of Rescarch Fundamental Care Online, 7(4), 3317-3326. Recuperado de http://www.seer.unirio.br/index.php/cuidadofundamental/ article/view/3911/pdf_1713

Oliveira, E. B., Silva, A. V., Perez Junior, E. F., Costa, H. F., Nascimento, L. P., \& Souza, L. A. M. (2013). Fatores de risco psicossocial em terapia intensiva neonatal: repercussões para a saúde do enfermeiro. Revista de Enfermagem da UERJ, 21(4), 490-495. Recuperado de https://www.e-publicacoes.uerj. br/index.php/enfermagemuerj/article/view/10020.

Oliveira, I. C. L., Cavalcante, M. L. S. N., Aires, S. F., Freitas, R. J. M., Silva, B. V., Marinho, D. M. F., \& Carvalho, R. E. F. L. (2018). Cultura de segurança: percepção dos profissionais de saúde em um hospital mental. Revista Brasileira de Enfermagem, 71(Suppl. 5), 2316-2322. doi: https://dx.doi. org/10.1590/0034-7167-2018-0125.

Rodrigues, C. M. L., Faiad, C., \& Facas, E. P. (2020). Fatores de risco e riscos psicossociais no trabalho: definição e implicações. Psicologia: Teoria e Pesquisa, 36(spe), 1-9. doi: https://doi.org/10.1590/0102.3772e36nspe19.

Rissi, V., Monteiro, J. K., \& Ferreira, V. R. T. (2020). Riscos psicossociais no trabalho em programas de pós-graduação stricto sensu públicos e privados. Trabalho En(Cena), 5(1), 227-247. doi: https://doi.org/10.20873/ 2526-1487V5N1P227.

Roncoleta, L. M., Jorge, I. M. P., Maríngolo, L. P., Justino, M. F., \& Silva-e-Dutra, F.C. M. (2019). Fatores psicossociais e condições ambientais: sua influência na capacidade para o trabalho na área da assistência social. Rev Bras Med Trab., 17(3):335-345. Recuperado de https://www.rbmt.org.br/details/470/pt-BR/fatores-psicossociais-e-condicoes-ambientais--sua-influencia-na-capacidade-para-0-trabalho-na-area-da-assistencia-social.

Scozzafave, M. C. S., Leal, L. A., Soares, M. I., \& Henriques, S. H. (2019). Riscos psicossociais relacionados ao enfermeiro no hospital psiquiátrico e estratégias de gestão. Revista Brasileira de Enfermagem, 72(4), 834-840. doi: https://dx.doi.org/10.1590/0034-7167-2017-0311.

Serafim, A.C., Campos, I. C. M., Cruz, R. M. \& Rabuske, M. M. (2012). Riscos Psicossociais e Incapacidade do Servidor Público: Um Estudo de Caso. Paideia, 32(3), 686-705. Recuperado de https://www.scielo.br/pdf/pcp/ v32n3/v32n3a13.pdf.

Silva, A. M., \& Guimarães, L. A. M. (2016). Occupational Stress and Quality of Life in Nursing. Paidéia (Ribeirão Preto), 26(63), 63-70. doi: https://doi. org/10.1590/1982-43272663201608.
Silva, L. A., Fritsch, J. N., Dalri, R. C. M. B., Giulena, R. L., Silveira, S. E., Evangelista, R. A., Pelazza, B. B., \& Maia, L. G. (2016). Riscos ocupacionais e adoecimentos entre professores da rede municipal de ensino. Journal Health NPEPS, 1(2), 178-196. Recuperado de https://periodicos.unemat. br/index.php/jhnpeps/article/view/1586.

Silva, S. C. P. S., Nunes, M. A. P., Santana, V. R., Reis, F. P., Machado Neto, J., \& Lima, S. 0. (2015). A síndrome de burnout em profissionais da Rede de Atenção Primária à Saúde de Aracaju, Brasil. Ciência \& Saúde Coletiva, 20(10), 3011-3020. doi: https://doi.org/10.1590/1413812320152010.19912014

Sobral, R. C., Stephan, C., Bedin-Zanatta, A., Lucca, S.R. (2018). Burnout e a organização do trabalho na Enfermagem. Rev Bras Med Trab., 16(1), 44-52. Recuperado de http://rbmt.org.br/details/292/pt-BR.

Sousa, K. H. J. F., Zeitoune, R. C. G., Portela, L. F., Tracera, G. M. P., Moraes, K. G., \& Figueiró, R. F. S. (2020). Fatores associados aos riscos de adoecimento da equipe de enfermagem no trabalho em instituição psiquiátrica. Revista Latino-Americana de Enfermagem, 28, e3235. doi: https://doi. org/10.1590/1518-8345.3454.3235.

Souza, E. R., Minayo, M. C. S., Silva, J. G., \& Pires, T. 0. (2012). Fatores associados ao sofrimento psíquico de policiais militares da cidade do Rio de Janeiro, Brasil. Cadernos de Saúde Pública, 28(7), 1297-1311. Recuperado de https://scielosp.org/article/csp/2012.v28n7/1297-1311/.

Souza, N. V. D. 0., Pires, A. S., Gonçalves, F. G. A., Cunha, L. S., Ribeiro, L. V., Vieira, R. S. (2014). Riscos ocupacionais e agravos à saúde dos trabalhadores em uma unidade ambulatorial especializada. Revista Mineira de Enfermagem, 18(4), 923-930. Recuperado de https://cdn.publisher.gn1. link/reme.org.br/pdf/v18n4a12.pdf

World Health Organization - WHO (2015). Mental health atlas 2014. World Health Organization. Recuperado de https://apps.who.int/iris/handle/10665/178879. 